\title{
A RIGOROUS REAL TIME FEYNMAN PATH INTEGRAL
}

\author{
KEN LOO \\ This work partially fulfills the author's Ph.D. thesis requirements \\ under the guidance of Professor James Thurber at Purdue University. \\ This work was supported by the Purdue research foundation.
}

\begin{abstract}
Using improper Riemann integrals, we will formulate a rigorous version of the real-time, time-sliced Feynman path integral for the $L^{2}$ transition probability amplitude. We will do this for nonvector potential Hamiltonians with potential which has at most a finite number of discontinuities and singularities. We will also provide a Nonstandard Analysis version of our formulation.
\end{abstract}

1. Introduction and Notations. In this paper, we will formulate a rigorous version of the real-time, time-sliced Feynman path integral for the $L^{2}$ transition probability amplitude

$$
\left\langle\phi^{*}, \exp \left(\frac{-i t \bar{H}}{\hbar}\right) \psi\right\rangle_{L^{2}}=\int_{\mathbb{R}^{n}} \phi(\vec{x})\left[\exp \left(\frac{-i t \bar{H}}{\hbar}\right) \psi\right](\vec{x}) d \vec{x},
$$

where $\phi, \psi \in L^{2}, H=\frac{-\hbar^{2}}{2 m} \Delta+V(\vec{x})$ is essentially self-adjoint, $\bar{H}$ is the closure of $H$, and $\phi, \psi, V$ each carries at most a finite number of singularities and discontinuities. In flavor of physics literature, we will formulate the Feynman path integral with improper Riemann integrals. In hope that with further research we can formulate a rigorous polygonal path integral, we will also provide a Nonstandard Analysis version of the Feynman path integral. Using Nonstandard Analysis is not essential to our formulation, and the idea of using Nonstandard Analysis on the Feynman path integral is not a new concept. For readers interested in Nonstandard Analysis, and its applications to Feynman path integrals, see [1], [10], [13], [19], [22], and references within. We will assume that the reader is familiar with Nonstandard Analysis.

In physics, the Feynman path integral is formulated on the propagator and it is formally given by (see [11], [14], and [21])

$$
K_{t}\left(\vec{x}, \vec{x}_{0}\right)=\lim _{k \rightarrow \infty} w_{n, k} \int_{\mathbb{R}^{(k-1) n}} \exp \left[\frac{i \epsilon}{\hbar} S_{k}\left(\vec{x}, \ldots \vec{x}_{o}\right)\right] d \vec{x}_{1} \ldots d \vec{x}_{k-1},
$$

The author would like to give thanks to his Ph.D. thesis committee: Daniel Gottlieb, Leonard Lipshitz, Herman Rubin, and James Thurber. Also, special thanks to Patricia Bauman. 
where

$$
\begin{aligned}
& w_{n, k}=\left(\frac{m}{2 i \pi \hbar \epsilon}\right)^{\frac{n k}{2}}, \epsilon=\frac{t}{k}, \\
& S_{k}\left(\vec{x}=\vec{x}_{k}, \ldots, \vec{x}_{0}\right)=\sum_{j=1}^{k}\left[\frac{m}{2}\left(\frac{\vec{x}_{j}-\vec{x}_{j-1}}{\epsilon}\right)^{2}-V\left(\vec{x}_{j}\right)\right] .
\end{aligned}
$$

and all integrals are improper Riemann integrals.

In mathematics, there is a there is a rigorous time-sliced Feynman path integral for the wave function (see [5] and [18])

$$
\begin{aligned}
& {\left[\exp \left(\frac{-i t \bar{H}}{\hbar}\right) \psi\right](\vec{x})=} \\
& \text { l.i.m }{ }_{k \rightarrow \infty} w_{n, k} \int_{\mathbb{R}^{k n}} \exp \left[\frac{i \epsilon}{\hbar} S_{k}\left(\vec{x}, \ldots, \vec{x}_{o}\right)\right] \psi\left(\vec{x}_{0}\right) d \vec{x}_{0} \ldots d \vec{x}_{k-1} .
\end{aligned}
$$

where the integrals in (1.4) are improper Lebesgue integrals and their convergence is in the $L^{2}$ norm. Other popular rigorous versions of the Feynman path integral are the Wiener integral(see [7], [8], [12], [16], and [18]), generalization of Fresnel integrals(see [2]), and Henstock integrals(see [15]). For a more detailed exposition and further references, see [2] and [3].

Our main concern in this paper is to provide a rigorous version of (1.2) for the transition probability amplitude given in (1.1) by using (1.4). We will show that for any essentially self-adjoint Hamiltonian with potential that carries a finite number of singularities and discontinuities and for any $\phi, \psi \in L^{2}$ which also has a finite number of singularities and discontinuities the following holds

$$
\begin{aligned}
& \int_{\mathbb{R}^{n}} \phi(\vec{x})\left[\exp \left(\frac{-i t \bar{H}}{\hbar}\right) \psi\right](\vec{x}) d \vec{x}= \\
& \lim _{k \rightarrow \infty} w_{n, k} \int_{\mathbb{R}^{(k+1) n}} \phi\left(\vec{x}_{k}\right) \exp \left[\frac{i \epsilon}{\hbar} S_{k}\left(\vec{x}_{k}, \ldots, \vec{x}_{0}\right)\right] \psi\left(\vec{x}_{0}\right) d \vec{x}_{0} \ldots d \vec{x}_{k} .
\end{aligned}
$$

In the last line of (1.5), the integral is an improper Riemann integral over $\mathbb{R}^{(k+1) n}$.

A trivial application of Nonstandard Analysis on the $k$ limit in (1.5) yields

$$
\begin{aligned}
& \int_{\mathbb{R}^{n}} \phi(\vec{x})\left[\exp \left(\frac{-i t \bar{H}}{\hbar}\right) \psi\right](\vec{x}) d \vec{x}= \\
& \operatorname{st}\left\{w_{n, \omega} \int_{{ }^{*}(\omega+1) n} \phi\left(\vec{x}_{\omega}\right) \exp \left[\frac{i \epsilon}{\hbar} S_{\omega}\left(\vec{x}_{\omega}, \ldots, \vec{x}_{0}\right)\right] \psi\left(\vec{x}_{0}\right) d \vec{x}_{0} \ldots d \vec{x}_{\omega}\right\},
\end{aligned}
$$

where the integral in the last line of (1.6) is a $*$-transformed improper Riemann integral over ${ }^{*} \mathbb{R}^{(\omega+1) n}$, and $\omega \in * \mathbb{N}-\mathbb{N}$.

The main idea in the proof of (1.5) is the following. For simplicity, suppose $f(x) \in L^{2}(\mathbb{R}), g(x, y) \in L^{2}(\mathbb{R} \times \mathbb{R})$ are such that they are bounded and continuous. Further, suppose that both

$$
\begin{aligned}
& h(x)=\int_{-a}^{b} g(x, y) d y \\
& p(x)=\text { l.i.m } \mathrm{m}_{a, b \rightarrow \infty} \int_{-a}^{b} g(x, y) d y
\end{aligned}
$$


are in $L^{2}(\mathbb{R})$ as a function of $x$. In (1.7), we take the integral to be Lebesgue integrals and the limits are taken independent of each other. Notice that for $p(x)$, we can interpret the integral as an improper Lebesgue integral with convergence in the $L^{2}$ topology. Let us denote $\chi_{[-c, d]}$ to be the characteristic function on $[-c, d]$. Schwarz's inequality then implies

$$
\begin{aligned}
& \left|\int_{\mathbb{R}} f(x) p(x)-\int_{-c}^{d} \int_{-a}^{b} f(x) g(x, y) d x d y\right| \leq \\
& \|f\|_{2}\|p-h\|_{2}+\left\|f-\chi_{[-c, d]} f\right\|_{2}\|h\|_{2} \rightarrow 0 .
\end{aligned}
$$

Thus, we can write

$$
\int_{\mathbb{R}} f(x) p(x)=\lim _{a, b, c, d \rightarrow \infty} \int_{-c}^{d} \int_{-a}^{b} f(x) g(x, y) d x d y,
$$

where the limits are all taken independent of each other. Since $f$ and $g$ are bounded and continuous, the Lebesgue integral over $[-a, b] \times[-c, d]$ in (1.9) can be replaced by a Riemann integral. Since the limits are taken independent of each other, we can then interpret the right hand-side of (1.9) as an improper Riemann integral. If $f$ and $g$ carry singularities and discontinuities, care must be taken in the region of integration so that the replacement of Lebesgue integral with Riemann integrals can be done.

We now set some notations to deal with $n$-dimensional integrations, singularities and discontinuities. Let $k \in \mathbb{N}$ and $0 \leq l \leq k$. We will denote the interior of the lth box by

$$
A^{l}=\left(-a_{1}^{l}, b_{1}^{l}\right) \times \cdots \times\left(-a_{n}^{l}, b_{n}^{l}\right),
$$

for positive and large $a$ 's and $b$ 's. Let $K=\left\{\vec{y}_{1} \ldots \vec{y}_{p}\right\}$ be the set of discontinuous and singular points of $\phi, \psi$ and $V$. For each $\vec{y}_{q}=\left(y_{1}^{q}, \ldots, y_{n}^{q}\right) \in K$, denote the $l$ th box centered at $\vec{y}_{q}$ by

$$
B_{q}^{l}=\left(y_{1}^{q}-\frac{1}{c_{1}^{q, l}}, y_{1}^{q}+\frac{1}{d_{1}^{q, l}}\right) \times \cdots \times\left(y_{n}^{q}-\frac{1}{c_{n}^{q, l}}, y_{n}^{q}+\frac{1}{d_{n}^{q, l}}\right)
$$

for positive and large $c$ 's and $d$ 's. Let

$$
C^{l}=A^{l}-\left\{\bigcup_{q=1}^{p} B_{q}^{l}\right\} .
$$

For arbitrary large $a^{\prime}$ 's, $b^{\prime} \mathrm{s}, c^{\prime}$ 's, and $d^{\prime}$ 's, $C^{l}$ is a box which encloses the set $K$ and at each point of $K$, a small box centered at that point is taken out. Associated with $C^{l}$ is a set of indices

$$
\begin{array}{r}
\left\{j_{l}\right\}=\left\{a_{1}^{l}, \ldots, a_{n}^{l}, b_{1}^{l}, \ldots, b_{n}^{l}, c_{1}^{1, l}, \ldots, c_{n}^{1, l}, \ldots, c_{1}^{p, l}, \ldots, c_{n}^{p, l}\right. \\
\left.d_{1}^{1, l}, \ldots, d_{n}^{1, l}, \ldots, d_{1}^{p, l}, \ldots, d_{n}^{p, l}\right\}
\end{array}
$$


We will denote by $\left\{j_{l}\right\} \rightarrow \infty$ to mean

$$
\begin{aligned}
& a_{1}^{l}, \ldots, a_{n}^{l}, b_{1}^{l}, \ldots, b_{n}^{l}, c_{1}^{1, l}, \ldots, c_{n}^{1, l}, \ldots, c_{1}^{p, l}, \ldots, c_{n}^{p, l}, \\
& d_{1}^{1, l}, \ldots, d_{n}^{1, l}, \ldots, d_{1}^{p, l}, \ldots, d_{n}^{p, l} \rightarrow \infty
\end{aligned}
$$

where all indices goes to infinity independent of each other. Notice that as $\left\{j_{l}\right\} \rightarrow$ $\infty$, we recover $\mathbb{R}^{n}$ a.e. from $C^{l}$. We will denote by $\chi_{\left\{j_{l}\right\}}$ the characteristic function on $C^{l}$. Notice that for $f \in L^{2}\left(\mathbb{R}^{n}\right)$,

$$
\text { l.i. } \mathrm{m}_{\left\{j_{l}\right\} \rightarrow \infty} \chi_{\left\{j_{l}\right\}} f=f \quad \text { a.e. }
$$

Lastly, let us write

$$
D_{\left\{J_{0}^{k}\right\}}=C^{0} \times \cdots \times C^{k} .
$$

Associated with $D_{\left\{J_{0}^{k}\right\}}$ is a set of indices

$$
\left\{J_{0}^{k}\right\}=\bigcup_{l=0}^{k}\left\{j_{l}\right\}
$$

and as before, we will use the notation $\left\{J_{0}^{k}\right\} \rightarrow \infty$ to mean

$$
\left\{j_{0}\right\} \rightarrow \infty, \ldots,\left\{j_{k}\right\} \rightarrow \infty
$$

where the indices are taken to infinity independent of each other.

From here on, we will assume that $\phi, \psi \in L^{2}$ and $V$ are such that they have at most a finite number of singularities and discontinuities and the set of those points are denoted as $K=\left\{\vec{y}_{1} \ldots \vec{y}_{p}\right\}$. Finally, we will denote by $\int_{r O}$ to be Riemann or improper Riemann integration over the region $O$ and $\int_{O}$ to be Lebesgue integration over the region $O$.

2. Feynman Path Integrals. The standard derivation of (1.4) is via the Trotter product formula (see [5], [16], and [18]) which says that for any essentially selfadjoint $H=H_{0}+V$ with $H_{0}=\frac{-\hbar^{2}}{2 m} \Delta$ and any $\psi \in L^{2}$,

$$
\exp \left(\frac{-i t \bar{H}}{\hbar}\right) \psi=\text { l.i.m. } k \rightarrow \infty\left\{\exp \left(\frac{-i t V}{k \hbar}\right) \exp \left(\frac{-i t H_{0}}{k \hbar}\right)\right\}^{k} \psi
$$

Thus, we have the following

Lemma 2.1. Suppose $H=H_{0}+V$ is essentially self-adjoint. Let $\psi, \phi \in L^{2}$, then

$$
\begin{aligned}
& \int_{\mathbb{R}^{n}} \phi(\vec{x})\left[\exp \left(\frac{-i t \bar{H}}{\hbar}\right) \psi\right](\vec{x}) d \vec{x}= \\
& \lim _{k \rightarrow \infty} \int_{\mathbb{R}^{n}} \phi(\vec{x})\left[\left\{\exp \left(\frac{-i t V}{k \hbar}\right) \exp \left(\frac{-i t H_{0}}{k \hbar}\right)\right\}^{k} \psi\right](\vec{x}) d \vec{x} .
\end{aligned}
$$


Proof. The proof is just an application of (2.1) and Schwarz's inequality.

It is well known that(see [5] and [18]) for $\nu \in L^{1}\left(\mathbb{R}^{n}\right) \bigcap L^{2}\left(\mathbb{R}^{n}\right)$,

$$
\left[\exp \left(\frac{-i \epsilon H_{0}}{\hbar}\right) \nu\right]\left(\vec{x}_{1}\right)=\left(\frac{m}{2 i \pi \hbar \epsilon}\right)^{\frac{n}{2}} \int_{\mathbb{R}^{n}} \exp \left[\frac{i m \epsilon}{2 \hbar}\left(\frac{\vec{x}_{1}-\vec{x}_{0}}{\epsilon}\right)^{2}\right] \nu\left(\vec{x}_{0}\right) d \vec{x}_{0},
$$

and that the operator $\exp \left(\frac{-i \epsilon H_{0}}{\hbar}\right)$ is unitary. Thus, we can write

$$
\begin{aligned}
& {\left[\exp \left(\frac{-i \epsilon H_{0}}{\hbar}\right) \psi\right]\left(\vec{x}_{1}\right)=\left[\exp \left(\frac{-i \epsilon H_{0}}{\hbar}\right)\left[1 . i \cdot \mathrm{m}_{\left\{j_{0}\right\} \rightarrow \infty} \chi_{\left\{j_{0}\right\}} \psi\right]\right]\left(\vec{x}_{1}\right)=} \\
& \text { l.i.m }\left\{j_{0}\right\} \rightarrow \infty\left[\exp \left(\frac{-i \epsilon H_{0}}{\hbar}\right) \chi_{\left\{j_{0}\right\}} \psi\right]\left(\vec{x}_{1}\right)= \\
& \text { l.i.m } \mathrm{m}_{\left\{j_{0}\right\} \rightarrow \infty}\left(\frac{m}{2 i \pi \hbar \epsilon}\right)^{\frac{n}{2}} \int_{C^{0}} \exp \left[\frac{i m \epsilon}{2 \hbar}\left(\frac{\vec{x}_{1}-\vec{x}_{0}}{\epsilon}\right)^{2}\right] \psi\left(\vec{x}_{0}\right) d \vec{x}_{0} .
\end{aligned}
$$

Notice that by construction of the region $C^{0}, \psi$ is bounded and continuous on $C^{0}$, hence the Lebesgue integral in the last line of (2.4) can be replaced by a Riemann integral.

For notation convenience, we will denote

$$
\begin{aligned}
& \rho\left(\vec{x}_{k},\left\{J_{0}^{k-1}\right\}\right)= \\
& w_{n, k} \int_{D_{\left\{J_{0}^{k-1}\right\}}} \exp \left[\frac{i t}{(k+1) \hbar} S_{k}\left(\vec{x}_{k}, \ldots, \vec{x}_{o}\right)\right] \psi\left(\vec{x}_{0}\right) d \vec{x}_{0} \ldots d \vec{x}_{k-1} \text {, } \\
& T=\exp \left(\frac{-i t V}{k \hbar}\right) \exp \left(\frac{-i t H_{0}}{k \hbar}\right), \quad T^{k}=\left\{\exp \left(\frac{-i t V}{k \hbar}\right) \exp \left(\frac{-i t H_{0}}{k \hbar}\right)\right\}^{k} .
\end{aligned}
$$

Lemma 2.2. Suppose $H=H_{0}+V$ is essentially self-adjoint. Let $\psi \in L^{2}$, then for $k \in \mathbb{N}$ the following holds

$$
\begin{aligned}
& \left\{\exp \left(\frac{-i t V}{k \hbar}\right) \exp \left(\frac{-i t H_{0}}{k \hbar}\right)\right\}^{k} \psi= \\
& \operatorname{li}_{\left\{J_{0}^{k-1}\right\} \rightarrow \infty} w_{n, k} \int_{D_{\left\{J_{0}^{k-1}\right\}}} \exp \left[\frac{i \epsilon}{\hbar} S_{k}\left(\vec{x}_{k}, \ldots, \vec{x}_{o}\right)\right] \psi\left(\vec{x}_{0}\right) d \vec{x}_{0} \ldots d \vec{x}_{k-1} \text {. }
\end{aligned}
$$

Proof. We will proof (2.6) by induction. Suppose $k=2$, then (2.4) implies

$$
\begin{aligned}
& T^{2} \psi=T\left\{\operatorname{li}_{\left\{J_{0}^{0}\right\} \rightarrow \infty} \rho\left(\vec{x}_{1},\left\{J_{0}^{0}\right\}\right)\right\}= \\
& \exp \left(\frac{-i t V}{2 \hbar}\right) \exp \left(\frac{-i t H_{0}}{2 \hbar}\right)\left\{\operatorname{li} \mathrm{m}_{\left\{j_{1}\right\} \rightarrow \infty} \chi_{\left\{j_{1}\right\}}\left[\operatorname{lid}_{\left\{J_{0}^{0}\right\} \rightarrow \infty} \rho\left(\vec{x}_{1},\left\{J_{0}^{0}\right\}\right)\right]\right\} .
\end{aligned}
$$


Since multiplication by a characteristic function, $\exp \left(\frac{-i t V}{2 \hbar}\right)$, and $\exp \left(\frac{-i t H_{0}}{2 \hbar}\right)$ are all continuous operators from $L^{2}$ to $L^{2}$, we can take the $L^{2}$ limits in (2.7) outside of the operators and we can do this in any order we wish. Hence, (2.6) is true for $k=2$. Assuming (2.6) to be true for $\mathrm{k}$, then

$$
\begin{aligned}
& T^{k+1} \psi=\exp \left(\frac{-i t V}{(k+1) \hbar}\right) \times \\
& \exp \left(\frac{-i t H_{0}}{(k+1) \hbar}\right)\left\{\lim _{\left\{j_{k}\right\} \rightarrow \infty} \chi_{\left\{j_{k}\right\}}\left[\lim _{\left\{J_{0}^{k-1}\right\} \rightarrow \infty} \rho\left(\vec{x}_{k},\left\{J_{0}^{k-1}\right\}\right)\right]\right\} .
\end{aligned}
$$

By the same reasoning as for the case of $k=2$, we can take all the $L^{2}$ limits in (2.8) outside of the operators and we can do this in any order we wish. Hence, (2.6) is true for all $k \in \mathbb{N}$.

Proposition 2.3. Suppose $H=H_{0}+V$ is essentially self-adjoint. Let $\psi, \phi \in L^{2}$, then for all $k \in \mathbb{N}$ the following is true

$$
\begin{aligned}
& \int_{\mathbb{R}^{n}} \phi(\vec{x})\left[\left\{\exp \left(\frac{-i t V}{k \hbar}\right) \exp \left(\frac{-i t H_{0}}{k \hbar}\right)\right\}^{k} \psi\right](\vec{x}) d \vec{x}= \\
& w_{n, k} \int_{r \mathbb{R}^{(k+1) n}} \phi\left(\vec{x}_{k}\right) \exp \left[\frac{i \epsilon}{\hbar} S_{k}\left(\vec{x}_{k}, \ldots, \vec{x}_{0}\right)\right] \psi\left(\vec{x}_{0}\right) d \vec{x}_{0} \ldots d \vec{x}_{k} .
\end{aligned}
$$

Proof. Lemma 2.2 implies that

$$
\begin{aligned}
& \int_{\mathbb{R}^{n}} \phi(\vec{x})\left[\left\{\exp \left(\frac{-i t V}{k \hbar}\right) \exp \left(\frac{-i t H_{0}}{k \hbar}\right)\right\}^{k} \psi\right](\vec{x}) d \vec{x}= \\
& w_{n, k} \int_{\mathbb{R}^{n}}\left\{\operatorname{li} \mathrm{m}_{\left\{j_{k}\right\} \rightarrow \infty} \chi_{\left\{j_{k}\right\}} \phi\left(\vec{x}_{k}\right)\right\}\left\{\operatorname{li} \mathrm{m}_{\left\{J_{0}^{k-1}\right\} \rightarrow \infty} w_{n, k}\right.
\end{aligned}
$$

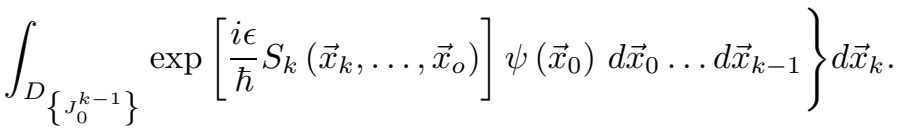

We now apply the idea in (1.8) and (1.9). Since all limits in (2.10) are taken independent of each other, we can use Schwarz's inequality and take all the $L^{2}$ limits outside of the integral as pointwise limits. Thus,

$$
\begin{aligned}
& \int_{\mathbb{R}^{n}} \phi(\vec{x})\left[\left\{\exp \left(\frac{-i t V}{k \hbar}\right) \exp \left(\frac{-i t H_{0}}{k \hbar}\right)\right\}^{k} \psi\right](\vec{x}) d \vec{x}= \\
& w_{n, k} \lim _{\left\{J_{0}^{k}\right\} \rightarrow \infty} \int_{D_{\left\{J_{0}^{k}\right\}}} \phi\left(\vec{x}_{k}\right) \exp \left[\frac{i \epsilon}{\hbar} S_{k}\left(\vec{x}_{k}, \ldots, \vec{x}_{o}\right)\right] \psi\left(\vec{x}_{0}\right) d \vec{x}_{0} \ldots d \vec{x}_{k} .
\end{aligned}
$$


By construction of $D_{\left\{J_{0}^{k}\right\}}$, the integrand $\phi\left(\vec{x}_{k}\right) \exp \left[\frac{i \epsilon}{\hbar} S_{k}\left(\vec{x}_{k}, \ldots, \vec{x}_{o}\right)\right] \psi\left(\vec{x}_{0}\right)$ in (2.12) is a bounded and continuous function on $D_{\left\{J_{0}^{k}\right\}}$. Hence, we can replace the Lebesgue integrals in (2.12) by Riemann integrals. Since all limits in (2.12) are taken independent of each other, we can interpret (2.12) as an improper Riemann integral.

We are now ready to proof (1.5).

Theorem 2.4. Suppose $H=H_{0}+V$ is essentially self-adjoint. Let $\psi, \phi \in L^{2}$. Furthermore, suppose that $\psi, \phi$, and $V$ has at most a finite number of singularities and discontinuities. With our previously defined notations, the following is true

$$
\begin{aligned}
& \int_{\mathbb{R}^{n}} \phi(\vec{x})\left[\exp \left(\frac{-i t \bar{H}}{\hbar}\right) \psi\right](\vec{x}) d \vec{x}= \\
& \lim _{k \rightarrow \infty} w_{n, k} \int_{r \mathbb{R}^{(k+1) n}} \phi\left(\vec{x}_{k}\right) \exp \left[\frac{i \epsilon}{\hbar} S_{k}\left(\vec{x}_{k}, \ldots, \vec{x}_{0}\right)\right] \psi\left(\vec{x}_{0}\right) d \vec{x}_{0} \ldots d \vec{x}_{k} .
\end{aligned}
$$

Proof. Follows from lemma 2.1 and proposition 2.3.

3. Nonstandard Feynman Path Integrals. A trivial application of Nonstandard Analysis on the $K$ limit in (2.2) will produce (1.6). It is our hope that with further research, a rigorous nonstandard polygonal path integral can be formulated.

Theorem 3.1. Suppose $H=H_{0}+V$ is essentially self-adjoint. Let $\psi, \phi \in L^{2}$. Furthermore, suppose that $\psi, \phi$, and $V$ has at most a finite number of singularities and discontinuities. With our previously defined notations, the following is true

$$
\begin{aligned}
& \int_{\mathbb{R}^{n}} \phi(\vec{x})\left[\exp \left(\frac{-i t \bar{H}}{\hbar}\right) \psi\right](\vec{x}) d \vec{x}= \\
& \operatorname{st}\left\{w_{n, \omega} \int_{r^{*} \mathbb{R}^{(\omega+1) n}} \phi\left(\vec{x}_{\omega}\right) \exp \left[\frac{i \epsilon}{\hbar} S_{\omega}\left(\vec{x}_{\omega}, \ldots, \vec{x}_{0}\right)\right] \psi\left(\vec{x}_{0}\right) d \vec{x}_{0} \ldots d \vec{x}_{\omega}\right\},
\end{aligned}
$$

where the integral in the last line of (3.1) is a *-transformed improper Riemann integral over ${ }^{*} \mathbb{R}^{(\omega+1) n}$, and $\omega \in * \mathbb{N}-\mathbb{N}$.

Proof. The nonstandard equivalent of lemma 2.1 is that for any $\omega \in{ }^{*} \mathbb{N}-\mathbb{N}$,

$$
\begin{aligned}
& \int_{\mathbb{R}^{n}} \phi(\vec{x})\left[\exp \left(\frac{-i t \bar{H}}{\hbar}\right) \psi\right](\vec{x}) d \vec{x}= \\
& s t\left\{\int_{\mathbb{R}^{n}} \phi(\vec{x})\left[\left\{\exp \left(\frac{-i t V}{\omega \hbar}\right) \exp \left(\frac{-i t H_{0}}{\omega \hbar}\right)\right\}^{\omega} \psi\right](\vec{x}) d \vec{x} .\right.
\end{aligned}
$$

After $*$-transforming proposition 2.3, Equation (3.1) follows from (3.2). 


\section{REFERENCES}

[1] S.Albeverio, J. Fenstat, R. Høegh-Krohn, and T. Lindstrøm, Nonstandard Methods in Stochastic Analysis and Mathematical Physics, Academic Press, 1986.

[2] S. Albeverio, and R. Høegh-Krohn, Mathematical Theory of Feynman Path Integrals, Lecture Notes in Mathematics, Vol. 523, Springer-Verlag, 1976.

[3] S. Albeverio et al., eds., Feynman Path Integrals, Lecture Notes in Physics Vol. 106, SpringerVerlag, 1979.

[4] W.O. Amrein, Non-Relativistic Quantum Dynamics, D. Reidel Publishing Company, 1981.

[5] J. Blank, P. Exner, M. Havliček, Hilber Space Operators in Quantum Physics, AIP press, 1994.

[6] N. Bogolubov, A. Logunov, A. Oksak, L. Todorov, General Principles of Quantum Field Theory, Kluwer Academic Publishers, 1990.

[7] R. Cameron, A Family of Integrals Serving to Connect The Wiener and Feynman Integrals, J. Math. and Phys. 39 (1961), 126-141.

[8] R. Cameron, The Ilstow and Feynman Integrals, J. Anal. Math 10 (1962/1963), 287-361.

[9] H. Cycon, R. Froese, W. Kirsch, B. Simon, Schrodinger Operators, Springer-Verlag, 1987.

[10] N. Cutland, Nonstandard Analysis and Its Applications, Cambridge University Press, 1988.

[11] R. Feynman, A. Hibbs, Quantum Mechanics and Path Integrals, McGraw-Hill, 1965.

[12] J. Glimm, and A. Jaffe, Quantum Physics, A Functional Integral Point of View, 2nd. Ed., Springer-Verlag, 1987.

[13] A.E.Hurd, P.A.Loeb, An Introduction to Nonstandard Real Analysis, Academic Press, 1985.

[14] H. Kleinert, Path Integrals in Quantum Mechanics, Statistics, and Polymer Physics, World Scientific, 1995.

[15] P. Muldowney, A General Theory of Integration in Function Spaces, Longman Scientific \& Technical, and John Wiley \& Sons, 1987.

[16] E. Nelson, Feynman Integrals and the Schrodinger Equation, J. Math. and Phys. 35 (1964), 332-343.

[17] E. Prugovečki, Quantum Mechanics in Hilbert Space, Academic Press, 1981.

[18] M. Reed, B. Simon, Functional Analysis I and II, Academic Press, 1980.

[19] A. Robinson, Nonstandard Analysis, Princeton University Press, 1996.

[20] G.Rosen, Formulations of Classical and Quantum Dynamical Theory, Academic Press, 1969.

[21] L. Schulman, Techniques and Applications of Path Integration, John Wiley and Son, 1981.

[22] K. Stroyan, W. Luxemburg, Introduction to the Theory of Infinitesimals, Academic Press, 1976.

[23] T. Kato, Perturbation Theory of Linear Operators, Springer-Verlag, 1995.

[24] K. Yosida, Functional Analysis, Springer-Verlag, 1995.

P.O. Box 9160, Portland, Oregon 97207

E-mail address: look@sdf.lonestar.org 